\title{
Tumor contrast enhancement using fluorescence lifetime: a new promise for image guided surgery (Conference Presentation) (Withdrawal Notice)
}

Anand Kumar, Rahul Pal, Lawrence Zukerberg, Kenneth Tanabe

Anand T. Kumar, Rahul Pal, Lawrence Zukerberg, Kenneth M. Tanabe, "Tumor contrast enhancement using fluorescence lifetime: a new promise for image guided surgery (Conference Presentation) (Withdrawal Notice)," Proc. SPIE 11625, Molecular-Guided Surgery: Molecules, Devices, and Applications VII, 116250T (15 March 2021); doi: 10.1117/12.2576446

SPIE. Event: SPIE BiOS, 2021, Online Only 


\section{Tumor contrast enhancement using fluorescence lifetime: a new promise for image guided surgery (Conference Presentation) (Withdrawal Notice)}

Anand T. Kumar, Rahul Pal, Lawrence Zukerberg, and Kenneth M. Tanabe

Massachusetts General Hospital (United States)

Proceedings Volume 1 1625, Molecular-Guided Surgery: Molecules, Devices, and Applications VII; $116250 T$ (2021) https://doi.org/10.1117/12.2560695

Event: Photonics West BiOS Digital Forum, 2021, Online Only

Online Publication Date: 5 March 2021

Withdrawn from Publication: 15 March 2021

Publisher's Note: This paper, originally published on 5 March 2021, was withdrawn per author request. 